\title{
Cluster linear generalized outer synchronization in community networks via pinning control with two different switch periods
}

\author{
Yuhong Liu ${ }^{1 *}$, Hui Li ${ }^{1}$, Qishui Zhong ${ }^{1}$ and Shouming Zhong ${ }^{2,3}$
}

\author{
"Correspondence: \\ yhliu1210@163.com \\ ${ }^{1}$ School of Aeronautics and \\ Astronautics, University of Electronic \\ Science and Technology of China, \\ Chengdu, 611731, China \\ Full list of author information is \\ available at the end of the article
}

\begin{abstract}
This study investigates the problem of cluster generalized outer synchronization in community networks via pinning control with two different switch periods. Several pinning controllers have been designed to achieve linear generalized outer synchronization. Using Lyapunov stability theory, sufficient linear generalized outer synchronization criteria for community networks are derived. Finally, numerical examples are presented to demonstrate the effectiveness of the theoretical results.
\end{abstract}

Keywords: community networks; linear generalized outer synchronization; pinning control

\section{然 Springer}

\section{Introduction}

Recently, complex networks have drawn increasing attention from researchers and engineers in virtue of its wide applications in many fields, such as World Wide Web, communication networks, social networks, neural networks, epidemic networks, traffic networks, etc. Lots of network models, such as weighted networks [1, 2], directed networks [3, 4], hierarchical networks [5], community networks [6-8] are introduced to explore the potential applications better. As is well known, the research on network synchronization is very important due to its potential applications in many fields including secure communication, laser transmission, image identification, information science, and so on [9-14]. In recent years, much literature reported the research results of network synchronization, and it has become a frontier issue [15-19]. As a result, different types of network synchronization have been put forward, for example, complete synchronization [20-22], phase synchronization $[23,24]$, projective synchronization $[25,26]$ and cluster synchronization $[27,28]$.

Furthermore, many real complex networks cannot synchronize themselves or synchronize with the desired orbits. Therefore, proper controllers should be designed to achieve the goals by adopting some control schemes, such as adaptive control [29], feedback control [30], observer-based control [31], impulsive control [32], intermittent control [33-35], pinning control $[36,37]$ and so on. As a matter of fact, there are many examples of relationships between different networks, which indicates that it is necessary and significant

(c) The Author(s) 2017. This article is distributed under the terms of the Creative Commons Attribution 4.0 International License (http://creativecommons.org/licenses/by/4.0/), which permits unrestricted use, distribution, and reproduction in any medium, provided you give appropriate credit to the original author(s) and the source, provide a link to the Creative Commons license, and indicate if changes were made. 
to investigate the dynamical systems between different networks. Recently, [38] investigated the synchronization between two unidirectionally coupled complex networks with identical topological structures. [39] discussed the synchronization between two complex dynamical networks with non-identical topological structures via using adaptive control method. [40] discussed adaptive projective synchronization between two complex networks with time-varying coupling delay. In the above papers, it is assumed that each node in drive-response networks has identical dynamics. Later, [41] studied the problem of generalized outer synchronization between two complex dynamical networks with different topologies and diverse node dynamics. Reference [42] discussed the linear generalized synchronization between two complex networks with the non-delay coupling and the same topological structure, each network has identical dynamics. However, detailed analysis of the linear generalized synchronization between two networks of different topological structures and time-varying coupling delay has not been attempted in [42].

Motivated by the above discussions, this paper investigates the problem of cluster linear generalized outer synchronization (CLGOS) in community networks via pinning control with two different switch periods. Several pinning controllers have been designed to achieve linear generalized outer synchronization. Using Lyapunov stability theory, sufficient linear generalized outer synchronization criteria for community networks are derived. Finally, numerical examples are presented to demonstrate the effectiveness of the theoretical results. Contributions of this paper can be summarized as follows:

- By adding adaptive semi-periodically intermittent controllers to a small fraction of nodes in response network, several sufficient conditions are derived based on the Lyapunov stability theory and strict mathematical proofs.

- Both community networks with identical nodes and non-identical nodes are investigated. Therefore, our proposed control schemes are more applicable technically.

The rest of the current paper is organized as follows. Section 2 introduces the problem formulation and some necessary definitions, lemmas, and hypotheses. Some sufficient conditions for the linear generalized outer synchronization are obtained in Section 3. Section 4 gives some numerical examples to demonstrate the effectiveness of our main results. Finally, Section 5 draws the conclusion.

Notation The superscripts $T$ and (-1) stand for matrix transposition and matrix inverse, respectively; $\mathbb{R}^{n}$ denotes the $n$-dimensional Euclidean space; $I_{l}$ means the l-dimensional identity matrix. The notation $X>Y(X \geq Y)$, where $X, Y$ are symmetric matrices, means that $X-Y$ is positive definite (positive semidefinite). $*$ denotes the term that is induced by symmetry. $\|\xi\|$ indicates the 2 -norm of a vector $\xi$, i.e., $\|\xi\|=\xi^{T} \xi$. $\operatorname{col}\left\{x_{1}, x_{2}, \ldots, x_{n}\right\}$ means $\left[x_{1}^{T}, x_{2}^{T}, \ldots, x_{n}^{T}\right]^{T}$ and $\operatorname{Sym}\{X\}$ means $X+X^{T}$. The shorthand notation $\operatorname{diag}\left\{M_{1}, M_{2}, \ldots, M_{n}\right\}$ denotes a block diagonal matrix with diagonal blocks being the matrices $M_{1}, M_{2}, \ldots, M_{n}$. $\lambda_{\min }(\cdot)$ and $\lambda_{\max }(\cdot)$ denote the smallest and largest eigenvalue of $\cdot$. The symbol $\otimes$ denotes the Kronecker product. Matrices, if their dimensions are not explicitly stated, are assumed to have appropriate dimensions for algebraic operations. 


\section{Problem formulation and preliminaries}

Consider the following complex networks with time-varying coupling delay consisting of $N$ nodes and s communities with $2 \leq s<N$

$$
\begin{aligned}
\dot{x}_{i}(t)= & f_{\varphi i}\left(x_{i}(t)\right)+c \sum_{k=1}^{s} \sum_{j \in V_{k}} a_{i j} \Gamma x_{j}(t) \\
& +c \sum_{k=1}^{s} \sum_{j \in V_{k}} b_{i j} \Gamma x_{j}(t-\tau(t)), \quad i \in 1,2, \ldots, N,
\end{aligned}
$$

where $x_{i} \in \mathbb{R}^{n}$ is the state variables of node $i$ in networks X. $f_{\varphi i}(\cdot): \mathbb{R}^{n} \longrightarrow \mathbb{R}^{n}$ is a continuously differentiable nonlinear function. $c$ is the coupling strength, and $\Gamma$ is an innercoupling matrix. $\tau(t)$ is the time-varying coupling delay satisfying $0 \leq \dot{\tau}(t) \leq \mu<1$. $A=\left(a_{i j}\right)_{N \times N} \in \mathbb{R}^{N \times N}$ and $B=\left(b_{i j}\right)_{N \times N} \in \mathbb{R}^{N \times N}$ are the outer-coupling matrices with the sum of each row being zero. If there is a connection from node $i$ to node $j(j \neq i)$, then the coupling $a_{i j}\left(b_{i j}\right) \neq 0$; otherwise, $a_{i j}\left(b_{i j}\right)=0(j \neq i)$, and the diagonal elements of matrix are defined as $a_{i i}=-\sum_{j=1, j \neq i}^{N} a_{i j}$ or $b_{i i}=-\sum_{j=1, j \neq i}^{N} b_{i j}$. The function $\varphi$ is defined as $\varphi$ : $\{1,2, \ldots, N\} \rightarrow\{1,2, \ldots, s\}$; if a node $i \in V_{k}$, then $\varphi_{i}=k ; V_{k}(k=1,2, \ldots, s)$ denotes the set of all nodes belong to the $s$ th community.

Consider the controller response complex dynamical network as follows:

$$
\begin{aligned}
\dot{y}_{i}(t)=\tilde{f}_{\varphi i}\left(y_{i}(t)\right)+c \sum_{k=1}^{s} \sum_{j \in V_{k}} a_{i j} \Gamma y_{j}(t) \\
+c \sum_{k=1}^{s} \sum_{j \in V_{k}} b_{i j} \Gamma y_{j}(t-\tau(t))+u_{i}(t), \quad i \in 1,2, \ldots, N,
\end{aligned}
$$

where $y_{i} \in \mathbb{R}^{n}$ is the response state variables of node $i$ in networks $Y . \tilde{f}_{\varphi i}(\cdot): \mathbb{R}^{n} \longrightarrow \mathbb{R}^{n}$ is a continuously differentiable nonlinear function. $u_{i}(t) \in \mathbb{R}^{n}(i=1,2, \ldots, N)$ are the nonlinear controllers ro be designed later, and the other parameters involved in system (2) all have the same meaning with the corresponding parameters in system (1).

Remark 2.1 The nonlinear vector-valued functions $f_{\varphi i}$ and $\tilde{f}_{\varphi i}$ can be identical or nonidentical.

Remark 2.2 There are no limitations for the division of the clusters, the number of nodes in each cluster and the connections between nodes.

Remark 2.3 All nodes within a cluster have the same dynamics, and the dynamics of the nodes in different clusters can be different.

Remark 2.4 The proposed approach on the case with undirected topology is similar to the one that on the case with directed topology. So in this paper the underlying topology is assumed to be undirected.

Suppose that the networks (2) will be controlled onto some desired inhomogeneous state as $\left\{y_{1}(t), \ldots, y_{m_{1}}(t)\right\} \rightarrow \phi_{1}(t),\left\{y_{m_{1}+1}(t), \ldots, y_{m_{2}}(t)\right\} \rightarrow \phi_{2}(t), \ldots,\left\{y_{m_{s-1}+1}(t), \ldots, y_{m_{s}}(t)\right\} \rightarrow$ 
$\phi_{s}(t)$, i.e., $\mathcal{M}=\left\{\left\{\phi_{1}(t), \ldots, \phi_{1}(t)\right\},\left\{\phi_{2}(t), \ldots, \phi_{2}(t)\right\}, \ldots,\left\{\phi_{s}(t), \ldots, \phi_{s}(t)\right\}\right\} \in \mathbb{R}^{n \times N}$ is desired cluster synchronization pattern under the pinning control.

Definition 2.1 Let $\phi_{\varphi_{i}}(t)=P x_{i}(t)+Q: \mathbb{R}^{n} \rightarrow \mathbb{R}^{n}(i=1,2, \ldots, N)$ be continuously differentiable vector maps. If $\varphi_{i} \neq \varphi_{j}, \phi_{\varphi_{i}} \neq \phi_{\varphi_{j}}$. Generalized outer synchronization between the drive-response networks are achieved if

$$
\lim _{t \rightarrow \infty}\left\|e_{i}(t)\right\|=\lim _{t \rightarrow \infty}\left\|y_{i}(t)-\phi_{\varphi_{i}}(t)\right\|=0, \quad i \in 1,2, \ldots, N
$$

where $P$ and $Q$ are constant matrices with proper dimension.

Assumption 2.1 Assuming that there are positive constants $L, \tilde{L}$ such that $f$ and $\tilde{f}$ satisfy the following inequalities:

$$
\begin{aligned}
& \left(y_{i}(t)-\phi_{\varphi_{i}}(t)\right)^{T}\left(f\left(y_{i}(t)\right)-f\left(\phi_{\varphi_{i}}(t)\right)\right) \leq L\left(y_{i}(t)-\phi_{\varphi_{i}}(t)\right)^{T} \Gamma\left(y_{i}(t)-\phi_{\varphi_{i}}(t)\right), \\
& \left(y_{i}(t)-\phi_{\varphi_{i}}(t)\right)^{T}\left(\tilde{f}\left(y_{i}(t)\right)-\tilde{f}\left(\phi_{\varphi_{i}}(t)\right)\right) \leq \tilde{L}\left(y_{i}(t)-\phi_{\varphi_{i}}(t)\right)^{T} \tilde{\Gamma}\left(y_{i}(t)-\phi_{\varphi_{i}}(t)\right),
\end{aligned}
$$

where $\Gamma$ and $\tilde{\Gamma}$ are positive definite matrix, $i=1,2, \ldots, N$. Here, $x$ and $y$ are time-varying vectors.

Lemma 2.1 For a diagonal matrix $D=\operatorname{diag}\{\underbrace{d_{1}, d_{2}, \ldots, d_{l}}_{i=\{1,2, \ldots, l\} \subseteq \bar{V}_{\varphi i}} 0,0, \ldots, 0\}$ with $d_{i}>0, \quad(i=$ $1,2, \ldots, l ; 1 \leq l \leq N)$ and a symmetric matrix $M \in \mathbb{R}^{N \times N}$, let $M-D=\left[\begin{array}{cc}E-\bar{D} & S \\ * & M_{l}\end{array}\right]$, where $M_{l}$ is the minor matrix of $M$ by removing its first $l(1 \leq l \leq N)$ row-column pairs, $E$ and $S$ are matrices with appropriate dimensions, $\bar{D}=\operatorname{diag}\left\{d_{1}, d_{2}, \ldots, d_{l}\right\}$. If $d_{i}>\lambda_{\max }\left(E-S M_{l}^{-1} S^{T}\right)$, then $M-D<0$ is equivalent to $M_{l}<0$.

Proof Let $\bar{D}=\operatorname{diag}\{\underbrace{d_{1}, d_{2}, \ldots, d_{l}}_{i=\{1,2, \ldots, l\} \subseteq \bar{V}_{\varphi i}}\}$. Using matrix decomposition, $M \in \mathbb{R}^{N \times N}$, let $M-D=$ $\left[\begin{array}{cc}E-\bar{D} & S \\ * & M_{l}\end{array}\right]$, where $M_{l}$ is the minor matrix of $M$ by removing its first $l(1 \leq l \leq N)$ row-column pairs, $E$ and $S$ are matrices with appropriate dimensions.

Using the Schur complement, it is easy to see that $M-D<0$ is equivalent to $M_{l}<0$. We only need to prove that if $M_{l}<0$, then $M-D<0$. When $d_{i}>0(i=1,2,3, \ldots, l)$ are sufficiently large such that $d_{i}>\lambda_{\max }\left(E-S M_{l}^{-1} S^{T}\right)$ hold, it is easy to see that $E-\bar{D}-S M_{l}^{-1} S^{T}<0$. Then, using the Schur complement, we can conclude that $M-D<0$, so the proof is finished.

Lemma 2.2 ([37]) Assume that $A, B$ are $N$ by $N$ Hermitian matrices. Let $\alpha_{1} \geq \alpha_{2} \geq \cdots \geq$ $\alpha_{N}, \beta_{1} \geq \beta_{2} \geq \cdots \geq \beta_{N}$ and $\gamma_{1} \geq \gamma_{2} \geq \cdots \gamma_{N}$ be eigenvalues of $A, B$ and $A+B$, respectively. Then one has $\alpha_{i}+\beta_{N} \leq \gamma_{i} \leq \alpha_{i}+\beta_{1}, i=1,2, \ldots, N$.

\section{Main results}

In this section, the CLGOS of the drive-response community networks (1) and (2) will be investigated in three cases. 
Case I. Assuming that the nodes dynamics in both community networks are identical, i.e., nonlinear vector-functions $f_{\varphi i}=\tilde{f}_{\varphi i}=f$ for all $1 \leq i \leq N$. Then the drive-response networks (1) and (2) can be written as

$$
\begin{aligned}
\dot{x}_{i}(t)= & f\left(x_{i}(t)\right)+c \sum_{k=1}^{s} \sum_{j \in V_{k}} a_{i j} \Gamma x_{j}(t) \\
& +c \sum_{k=1}^{s} \sum_{j \in V_{k}} b_{i j} \Gamma x_{j}(t-\tau(t)), \quad i \in 1,2, \ldots, N, \\
\dot{y}_{i}(t)= & f\left(y_{i}(t)\right)+c \sum_{k=1}^{s} \sum_{j \in V_{k}} a_{i j} \Gamma y_{j}(t) \\
& +c \sum_{k=1}^{s} \sum_{j \in V_{k}} b_{i j} \Gamma y_{j}(t-\tau(t))+u_{i}(t), \quad i \in 1,2, \ldots, N .
\end{aligned}
$$

In this subsection, the intermittent control with two different switched periods is considered. The sketch of such control strategy is given by Figure 1. As shown in Figure 1, $T_{1}$ and $T_{2}$ are two periods appearing alternately. $\eta_{1}\left(0<\eta_{1}<1\right)$ and $\eta_{2}\left(0<\eta_{2}<1\right)$ are called the rates of control duration in each control period. $\left(1-\eta_{1}\right) T_{1}$ and $\left(1-\eta_{2}\right) T_{2}$ are called non-feedback control widths in control periods $T_{1}$ and $T_{2}$, respectively. The rates of control duration may be different, i.e., $\eta_{1} \neq \eta_{2}$, while they are assumed to be the same in [21]. In this regards, the semi-periodically intermittent control scheme considered here is more general than [21].

We denote $\Xi_{1}^{m}=\left[m T, m T+\eta_{1} T_{1}\right]$ is the control width in period $T_{1}, \Xi_{2}^{m}=[m T+$ $\left.\eta_{1} T_{1}, m T+T_{1}\right]$ is the non-feedback control width in period $T_{1}, \Xi_{3}^{m}=\left[m T+T_{1}, m T+T_{1}+\right.$ $\left.\eta_{2} T_{2}\right]$ is the control width in period $T_{2}, \Xi_{4}^{m}=\left[m T+T_{1}+\eta_{2} T_{2},(m+1) T\right]$ the non-feedback control width in period $T_{2}$, where $m=0,1,2, \ldots$.

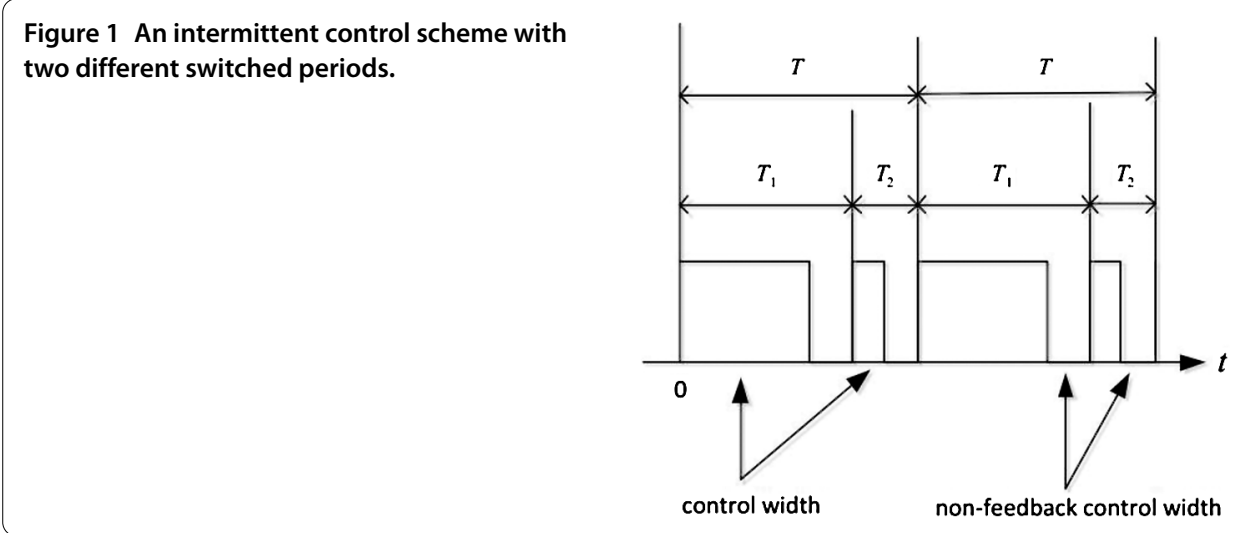


The adaptive semi-periodically intermittent controllers are defined as follows:

$$
u_{i}(t)=\left\{\begin{array}{l}
P f\left(x_{i}(t)\right)-f\left(\phi_{\varphi_{i}}(t)\right)+c \sum_{j=1}^{N} \tilde{p}_{i j} \Gamma y_{i}(t-\tau(t))-c \sum_{j=1}^{N} b_{i j} \Gamma e_{j}(t-\tau(t)) \\
\quad-c k_{i}(t) \Gamma e_{i}(t), \quad i \in \bar{V}_{\varphi i}, t \in \Xi_{1}^{m} \cup \Xi_{3}^{m}, \\
P f\left(x_{i}(t)\right)-f\left(\phi_{\varphi_{i}}(t)\right)+c \sum_{j=1}^{N} \tilde{p}_{i j} \Gamma y_{i}(t-\tau(t))-c \sum_{j=1}^{N} b_{i j} \Gamma e_{j}(t-\tau(t)), \\
\quad i \in \bar{V}_{\varphi i}, t \in \Xi_{2}^{m} \cup \Xi_{4}^{m}, \\
P f\left(x_{i}(t)\right)-f\left(\phi_{\varphi_{i}}(t)\right)+c \sum_{j=1}^{N} \tilde{p}_{i j} \Gamma y_{i}(t-\tau(t))-c \sum_{j=1}^{N} b_{i j} \Gamma e_{j}(t-\tau(t)), \\
\quad i \subsetneq \bar{V}_{\varphi i}, t \in \Xi_{1}^{m} \cup \Xi_{3}^{m}, \\
P f\left(x_{i}(t)\right)-f\left(\phi_{\varphi_{i}}(t)\right)+c \sum_{j=1}^{N} \tilde{p}_{i j} \Gamma y_{i}(t-\tau(t))-c \sum_{j=1}^{N} b_{i j} \Gamma e_{j}(t-\tau(t)), \\
\quad i \subsetneq \bar{V}_{\varphi i}, t \in \Xi_{2}^{m} \cup \Xi_{4}^{m},
\end{array}\right.
$$

where $\bar{V}_{\varphi i}$ denotes the set of the nodes in the $\varphi_{i}$ th community which have direct connections to the nodes in other communities and the updating laws

$$
\dot{\tilde{p}}_{i j}=-e_{i}^{T}(t) \Gamma y_{i}(t-\tau(t)), \quad i, j=1,2,3, \ldots, N
$$

and

$$
\dot{k}_{i}(t)= \begin{cases}\varepsilon_{i} e^{\beta t} e_{i}^{T}(t) \Gamma e_{i}(t), & t \in \Xi_{1}^{m} \cup \Xi_{3}^{m}, \\ 0, & t \in \Xi_{2}^{m} \cup \Xi_{4}^{m},\end{cases}
$$

where $\varepsilon_{i}\left(i \in \bar{V}_{\varphi i}\right)$ and $\beta$ are positive constants.

According to the definition of the coupling matrix $A, B$, it is easy to see that

$$
\left\{\begin{array}{l}
c \sum_{j=1}^{N} a_{i j} \Gamma Q=0, \\
c \sum_{j=1}^{N} b_{i j} \Gamma Q=0 .
\end{array}\right.
$$

Let $e_{i}(t)=y_{i}(t)-\phi_{\varphi_{i}}(t), g\left(e_{i}(t)\right)=f\left(y_{i}(t)\right)-f\left(\phi_{\varphi i}(t)\right), g(\cdot): \mathbb{R}^{n} \longrightarrow \mathbb{R}^{n}$ is a continuously differentiable nonlinear function. With the aid of equations (5)-(10), the error systems can be rewritten as

$$
\left\{\begin{aligned}
\dot{e}_{i}(t) & =g\left(e_{i}(t)\right)+c \sum_{j=1}^{N} a_{i j} \Gamma e_{j}(t)+c \sum_{j=1}^{N} \tilde{p}_{i j} \Gamma y_{i}(t-\tau(t))-c k_{i}(t) \Gamma e_{i}(t), \\
& i \in \bar{V}_{\varphi i}, t \in \Xi_{1}^{m} \cup \Xi_{3}^{m}, \\
\dot{e}_{i}(t) & =g\left(e_{i}(t)\right)+c \sum_{j=1}^{N} a_{i j} \Gamma e_{j}(t)+c \sum_{j=1}^{N} \tilde{p}_{i j} \Gamma y_{i}(t-\tau(t)), \\
\quad i & \in \bar{V}_{\varphi i}, t \in \Xi_{2}^{m} \cup \Xi_{4}^{m}, \\
\dot{e}_{i}(t) & =g\left(e_{i}(t)\right)+c \sum_{j=1}^{N} a_{i j} \Gamma e_{j}(t)+c \sum_{j=1}^{N} \tilde{p}_{i j} \Gamma y_{i}(t-\tau(t)), \\
& i \subsetneq \bar{V}_{\varphi i}, t \in \Xi_{1}^{m} \cup \Xi_{3}^{m}, \\
\dot{e}_{i}(t) & =g\left(e_{i}(t)\right)+c \sum_{j=1}^{N} a_{i j} \Gamma e_{j}(t)+c \sum_{j=1}^{N} \tilde{p}_{i j} \Gamma y_{i}(t-\tau(t)), \\
i & \bar{V}_{\varphi i}, t \in \Xi_{2}^{m} \cup \Xi_{4}^{m} .
\end{aligned}\right.
$$

Theorem 3.1 Suppose that Assumption 2.1 holds. Using the adaptive controllers and updated laws (7)-(9), then the response networks (6) can linear generalized synchronize with 
the drive networks (5) if there exist positive constants $\alpha>\beta>0$ such that the following conditions are satisfied:

$$
\begin{aligned}
& c<-\frac{\kappa}{\lambda_{\max }\left(\hat{A}_{l}\right)}, \\
& L \Gamma+\frac{\beta-\alpha}{2} I_{N}+\frac{1}{2(1-\mu)} I_{N}<0, \\
& \beta-\alpha \theta>0, \\
& \alpha \rho-a_{*}>0,
\end{aligned}
$$

where

$$
\begin{aligned}
& \kappa=\frac{1}{\lambda_{\min }(\Gamma)}\left(L\|\Gamma\|+\frac{\beta}{2}+\frac{1}{2(1-\mu)}\right), \\
& \theta=\left(1-\eta_{1}\right) \frac{T_{1}}{T}+\left(1-\eta_{2}\right) \frac{T_{2}}{T}, \quad \rho=\frac{\eta_{1} T_{1}+\eta_{2} T_{2}}{T}, \\
& \hat{A}=\frac{\operatorname{Sym}\{A\}}{2}, \quad a_{*}=\alpha-\beta,
\end{aligned}
$$

$\hat{A}_{l}$ is the minor matrix of $A$ by removing its first $l(1 \leq l \leq N)$ row-column pairs.

Proof Construct the following Lyapunov function candidate:

$$
V(t)=\sum_{i=1}^{4} V_{i}(t),
$$

where

$$
\begin{aligned}
& V_{1}(t)=\frac{1}{2} \sum_{i=1}^{N} e_{i}^{T}(t) e_{i}(t), \\
& V_{2}(t)=\frac{1}{2} \sum_{i=1}^{N} \sum_{j=1}^{N} c \tilde{p}_{i j}^{2}, \\
& V_{3}(t)=\frac{1}{2} \sum_{i \in \bar{V}_{\varphi i}} \exp (-\beta t) \frac{c\left(k_{i}(t)-k_{i}^{*}\right)^{2}}{\varepsilon_{i}}, \\
& V_{4}(t)=\frac{1}{2(1-\mu)} \sum_{i=1}^{N} \int_{t-\tau(t)}^{t} \tilde{e}_{j}^{T}(\theta) \tilde{e}_{j}(\theta) d \theta,
\end{aligned}
$$

where $k_{i}^{*}$ are sufficiently large positive constant to be determined. We write $\tilde{e}_{j}(\theta)=$ $\left(\tilde{e}_{1 j}(\theta), \tilde{e}_{2 j}(\theta), \ldots, \tilde{e}_{N j}(\theta)\right)^{T} \cdot \dot{\tilde{p}}_{i j}=-e_{i}^{T}(t) \Gamma y_{i}(t-\tau(t))$. 
When $t \in \Xi_{1}^{m} \cup \Xi_{3}^{m}$, differentiating $V(t)$ with respect to time along the solution of (11) yields

$$
\begin{aligned}
\dot{V}_{1}(t)= & \sum_{i=1}^{N} e_{i}^{T}(t) \dot{e}_{i}(t) \\
= & \sum_{i=1}^{N} e_{i}^{T}(t)\left[g\left(e_{i}(t)\right)+c \sum_{j=1}^{N} a_{i j} \Gamma e_{j}(t)+c \sum_{j=1}^{N} \tilde{p}_{i j} \Gamma y_{i}(t-\tau(t))\right] \\
& -c \sum_{i \in \bar{V}_{\varphi i}} e_{i}^{T}(t) k_{i}(t) \Gamma e_{i}(t), \\
\dot{V}_{2}(t)= & \sum_{i=1}^{N} \sum_{j=1}^{N} c \tilde{p}_{i j} \dot{\tilde{p}}_{i j}=-c \sum_{i=1}^{N} \sum_{j=1}^{N} \tilde{p}_{i j} e_{i}^{T}(t) \Gamma y_{i}(t-\tau(t)), \\
\dot{V}_{3}(t)= & c \sum_{i \in \bar{V}_{\varphi i}}\left(k_{i}(t)-k_{i}^{*}\right) e_{i}^{T}(t) \Gamma e_{i}(t)-\frac{\beta}{2} e^{-\beta t} \sum_{i \in \bar{V}_{\varphi i}} \frac{c\left(k_{i}(t)-k_{i}^{*}\right)^{2}}{\varepsilon_{i}}, \\
\dot{V}_{4}(t)= & \frac{1}{2(1-\mu)} \sum_{i=1}^{N} \tilde{e}_{j}^{T}(t) \tilde{e}_{j}(t)-\frac{1-\dot{\tau}(t)}{2(1-\mu)} \tilde{e}_{j}^{T}(t-\tau(t)) \tilde{e}_{j}(t-\tau(t)) .
\end{aligned}
$$

From (17)-(20), it is easy to see that

$$
\begin{aligned}
\dot{V}(t)= & \sum_{i=1}^{4} \dot{V}_{i}(t) \\
= & \sum_{i=1}^{N} e_{i}^{T}(t)\left[g\left(e_{i}(t)\right)+c \sum_{j=1}^{N} a_{i j} \Gamma e_{j}(t)+c \sum_{j=1}^{N} \tilde{p}_{i j} \Gamma y_{i}(t-\tau(t))\right] \\
& -c \sum_{i \in \bar{V}_{\varphi i}} e_{i}^{T}(t) k_{i}^{*} \Gamma e_{i}(t)-c \sum_{i=1}^{N} \sum_{j=1}^{N} \tilde{p}_{i j} e_{i}^{T}(t) \Gamma y_{i}(t-\tau(t)) \\
& -\frac{\beta}{2} e^{-\beta t} \sum_{i \in \bar{V}_{\varphi i}} \frac{c\left(k_{i}(t)-k_{i}^{*}\right)^{2}}{\varepsilon_{i}} \\
& +\frac{1}{2(1-\mu)} \sum_{i=1}^{N} \tilde{e}_{j}^{T}(t) \tilde{e}_{j}(t)-\frac{1-\dot{\tau}(t)}{2(1-\mu)} \tilde{e}_{j}^{T}(t-\tau(t)) \tilde{e}_{j}(t-\tau(t)) \\
\leq & \sum_{i=1}^{N} e_{i}^{T}(t)(L \Gamma+c \hat{A} \Gamma) e_{i}(t)+\frac{1}{2(1-\mu)} \sum_{i=1}^{N} \tilde{e}_{j}^{T}(t) \tilde{e}_{j}(t) \\
& -\frac{1-\dot{\tau}(t)}{2(1-\mu)} \tilde{e}_{j}^{T}(t-\tau(t)) \tilde{e}_{j}(t-\tau(t)) \\
& -c \sum_{i \in \bar{V}_{\varphi i}} e_{i}^{T}(t) k_{i}^{*} \Gamma e_{i}(t)-\beta V_{3}(t) \\
= & \sum_{i=1}^{N} e_{i}^{T}(t)\left(L \Gamma+c \hat{A} \Gamma+\frac{\beta}{2} I_{N}-c K^{*} \Gamma\right) e_{i}(t)+\frac{1}{2(1-\mu)} \sum_{i=1}^{N} \tilde{e}_{j}^{T}(t) \tilde{e}_{j}(t) \\
& -\frac{1-\dot{\tau}(t)}{2(1-\mu)} \tilde{e}_{j}^{T}(t-\tau(t)) \tilde{e}_{j}(t-\tau(t))-\beta\left(V_{1}(t)+V_{3}(t)\right) \\
&
\end{aligned}
$$




$$
\begin{aligned}
& \leq e^{T}(t)\left(L \Gamma+\frac{\beta}{2} I_{N}+\frac{1}{2(1-\mu)} I_{N}\right) e(t)+e^{T}(t)\left(\left(c \hat{A}-c K^{*}\right) \otimes \Gamma\right) e(t)-\beta V(t) \\
& \leq e^{T}(t)\left(\left(\kappa I_{N}+c \hat{A}-c K^{*}\right) \otimes \Gamma\right)-\beta V(t),
\end{aligned}
$$

where

$$
\begin{aligned}
& e(t)=\operatorname{col}\left\{e_{1}(t), e_{2}(t), \ldots, e_{n}(t)\right\}, \\
& K^{*}=\operatorname{diag}\{\underbrace{k_{1}^{*}, k_{2}^{*}, \ldots, k_{1}^{*}}_{i=\{1,2,3, \ldots, l\} \subseteq \bar{V}_{\varphi i}}, 0, \ldots, 0\}, \\
& \kappa=\frac{1}{\lambda_{\min }(\Gamma)}\left(L\|\Gamma\|+\frac{\beta}{2}+\frac{1}{2(1-\mu)}\right) .
\end{aligned}
$$

Let $\Omega=\kappa I_{N}+c\left(\hat{A}-K^{*}\right)=\left[\begin{array}{cc}E-K^{* *} & S \\ * & \Omega_{l}\end{array}\right]$, in which $\Omega_{l}$ is the minor matrix of $\Omega$ by removing its $l\left(l \in \bar{V}_{\varphi i}\right)$ row-column pairs, $E$ and $S$ are matrices with appropriate dimensions, $K^{* *}=$ $\operatorname{diag}\{\underbrace{c k_{1}, c k_{2}, \ldots, c k_{l}}\}$. It is obvious that $\Omega$ is symmetric. According to Lemma 2.1, we know $\underbrace{}_{i=\{1,2,3, \ldots, l\} \subseteq \bar{V}_{\varphi i}}$

that if one can select $k_{i}>\lambda_{\max }\left(E-S \Omega_{l}^{-1} S^{T}\right)$, then $\Omega<0$ is equivalent to $\Omega_{l}<0$. Based on Lemma 2.2 and the condition (12), we have $\lambda_{\max }\left(\Omega_{l}\right) \leq \kappa+c \lambda_{\max }\left(A_{l}\right)<0$, which implies that $\Omega_{l}<0$. Then we obtain

$$
\dot{V}(t) \leq-\beta V(t)
$$

When $t \in \Xi_{2}^{m} \cup \Xi_{4}^{m}$, differentiating $V(t)$ with respect to time along the solution of (11) and using the condition in (13) yields

$$
\begin{aligned}
\dot{V}(t)= & \sum_{i=1}^{4} \dot{V}_{i}(t) \\
= & \sum_{i=1}^{N} e_{i}^{T}(t)\left[g\left(e_{i}(t)\right)+c \sum_{j=1}^{N} a_{i j} \Gamma e_{j}(t)+c \sum_{j=1}^{N} \tilde{p}_{i j} \Gamma y_{i}(t-\tau(t))\right] \\
& -c \sum_{i=1}^{N} \sum_{j=1}^{N} \tilde{p}_{i j} e_{i}^{T}(t) \Gamma y_{i}(t-\tau(t))-\frac{\beta}{2} e^{-\beta t} \sum_{i \in \bar{\varphi}_{\varphi i}} \frac{c\left(k_{i}(t)-k_{i}^{*}\right)^{2}}{\varepsilon_{i}} \\
& +\frac{1}{2(1-\mu)} \sum_{i=1}^{N} \tilde{e}_{j}^{T}(t) \tilde{e}_{j}(t)-\frac{1-\dot{\tau}(t)}{2(1-\mu)} \tilde{e}_{j}^{T}(t-\tau(t)) \tilde{e}_{j}(t-\tau(t)) \\
\leq & e^{T}(t)\left(L \Gamma+\frac{\beta-\alpha}{2} I_{N}+\frac{1}{2(1-\mu)} I_{N}\right) e(t)+e^{T}(t)(c \hat{A} \otimes \Gamma) e(t) \\
& -\frac{\beta-\alpha}{2}\left(\sum_{i=1}^{N} e_{i}^{T}(t) e_{i}(t)+\sum_{i \in \bar{V}_{\varphi i}} \exp (-\beta t) \frac{c\left(k_{i}(t)-k_{i}^{*}\right)^{2}}{\varepsilon_{i}}\right) \\
\leq & (\alpha-\beta)\left(V_{1}(t)+V_{3}(t)\right) \\
\leq & (\alpha-\beta) V(t)=a_{*} V(t) .
\end{aligned}
$$


Then we have

$$
\dot{V}_{i}(t)= \begin{cases}-\beta V(t), & t \in \Xi_{1}^{m} \cup \Xi_{3}^{m}, \\ a_{*} V(t), & t \in \Xi_{2}^{m} \cup \Xi_{4}^{m} .\end{cases}
$$

From (24), it is easy to see that:

When $t \in \Xi_{1}^{m}$, i.e., $\frac{t-\eta_{1} T_{1}}{T}<m \leq \frac{t}{T}$

$$
\begin{aligned}
V(t) & \leq V(m T) \exp (-\beta(t-m T)) \\
& \leq V(0) \exp \left(-\beta m T+m \alpha\left(\left(1-\eta_{1}\right) T_{1}+\left(1-\eta_{2}\right) T_{2}\right)\right) \\
& \leq V(0) \exp \left((-\beta+\alpha \theta) t+\beta \eta_{1} T_{1}\right) .
\end{aligned}
$$

When $t \in \Xi_{2}^{m}$, i.e., $\frac{t-T_{1}}{T}<m \leq \frac{t-\delta_{1} T_{1}}{T}$,

$$
\begin{aligned}
V(t) & \leq V\left(m T+T_{1}\right) \exp \left(t-\left(m T+T_{1}\right)\right) \\
& \leq V(0) \exp \left((\alpha-\beta)\left(m T+T_{1}\right)-\alpha\left((m+1) \eta_{1} T_{1}+m \eta_{2} T_{2}\right)\right) \\
& \leq V(0) \exp \left(\left(a_{*}-\alpha \rho\right) t-\alpha\left(\eta_{1}-\eta_{2}\right) \frac{T_{1} T_{2}}{T}+a_{*}\left(1-\eta_{1}\right) T_{1}\right) .
\end{aligned}
$$

When $t \in \Xi_{3}^{m}$, i.e., $\frac{t-T_{1}-\eta_{2} T_{2}}{T}<m \leq \frac{t-T_{1}}{T}$,

$$
\begin{aligned}
V(t) & \leq V\left(m T+T_{1}\right) \exp \left(-\beta\left(t-\left(m T+T_{1}\right)\right)\right) \\
& \leq V(0) \exp \left(-\beta\left(m T+T_{1}\right)+\alpha\left((m+1)\left(1-\eta_{1}\right) T_{1}+m\left(1-\eta_{2}\right) T_{2}\right)\right) \\
& \leq V(0) \exp \left((-\beta+\alpha \theta)+\beta \eta_{2} T_{2}+\alpha \frac{\eta_{2}-\eta_{1}}{T} T_{1} T_{2}\right) .
\end{aligned}
$$

When $t \in \Xi_{4}^{m}$, i.e., $\frac{t}{T}<m+1 \leq \frac{t+T-T_{1}-\eta_{2} T_{2}}{T}$,

$$
\begin{aligned}
V(t) & \leq V((m+1) T) \exp \left(a_{*}(t-(m+1) T)\right) \\
& \leq V(0) \exp \left(a_{*}(m+1) T-(m+1) \alpha\left(\eta_{2} T_{2}+\eta_{1} T_{1}\right)\right) \\
& \leq V(0) \exp \left(\left(a_{*}-\alpha \rho\right) t+a_{*}\left(1-\eta_{2}\right) T_{2}\right) .
\end{aligned}
$$

Therefore, when $t \in \Xi_{1}^{m} \cup \Xi_{3}^{m}$, if $\beta-\alpha \theta>0$ is satisfied, one has $\lim _{t \rightarrow \infty} V(t)=0$; when $t \in$ $\Xi_{2}^{m} \cup \Xi_{4}^{m}$, if $\alpha \rho-a_{*}>0$ is satisfied, one has $\lim _{t \rightarrow \infty} V(t)=0$. The conclusion of Theorem 3.1 holds. This completes the proof.

Case II. Assume that the nodes dynamics in both community networks are nonidentical; in view of the special property, the adaptive semi-periodically intermittent con- 
trollers are defined as follows:

$$
\begin{aligned}
& u_{i}(t)=\left\{\begin{array}{l}
P f_{\varphi i}\left(x_{i}(t)\right)-\tilde{f}_{\varphi i}\left(\phi_{\varphi_{i}}(t)\right)+c \sum_{j=1}^{N} \tilde{p}_{i j} \Gamma y_{i}(t-\tau(t)) \\
\quad-c \sum_{j=1}^{N} b_{i j} \Gamma e_{j}(t-\tau(t))-c k_{i}(t) \Gamma e_{i}(t), \quad i \in \bar{V}_{\varphi i}, t \in \Xi_{1}^{m} \cup \Xi_{3}^{m}, \\
P f_{\varphi i}\left(x_{i}(t)\right)-\tilde{f}_{\varphi i}\left(\phi_{\varphi_{i}}(t)\right)+c \sum_{j=1}^{N} \tilde{p}_{i j} \Gamma y_{i}(t-\tau(t)) \\
\quad-c \sum_{j=1}^{N} b_{i j} \Gamma e_{j}(t-\tau(t)), \quad i \in \bar{V}_{\varphi i}, t \in \Xi_{2}^{m} \cup \Xi_{4}^{m}, \\
P f_{\varphi i}\left(x_{i}(t)\right)-\tilde{f}_{\varphi i}\left(\phi_{\varphi_{i}}(t)\right)+c \sum_{j=1}^{N} \tilde{p}_{i j} \Gamma y_{i}(t-\tau(t)) \\
-c \sum_{j=1}^{N} b_{i j} \Gamma e_{j}(t-\tau(t)), \quad i \subsetneq \bar{V}_{\varphi i}, t \in \Xi_{1}^{m} \cup \Xi_{3}^{m}, \\
P f_{\varphi i}\left(x_{i}(t)\right)-\tilde{f}_{\varphi i}\left(\phi_{\varphi_{i}}(t)\right)+c \sum_{j=1}^{N} \tilde{p}_{i j} \Gamma y_{i}(t-\tau(t))
\end{array}\right. \\
& \dot{k}_{i}(t)= \begin{cases}-c \sum_{j=1}^{N} b_{i j} \Gamma e_{j}(t-\tau(t)), \quad i \subsetneq \bar{V}_{\varphi i}, t \in \Xi_{2}^{m} \cup \Xi_{4}^{m}, \\
\varepsilon_{i} e^{\beta t} e_{i}^{T}(t) \Gamma e_{i}(t), & t \in \Xi_{1}^{m} \cup \Xi_{3}^{m}, \\
0, & t \in \Xi_{2}^{m} \cup \Xi_{4}^{m} .\end{cases}
\end{aligned}
$$

Theorem 3.2 Suppose that Assumption 2.1 holds. Using the adaptive controllers and updated laws (29)-(30), then the response networks (2) can linear generalized synchronize with the drive networks (1) if there exist positive constants $\alpha>\beta>0$ such that the following conditions are satisfied:

$$
\begin{aligned}
& c<-\frac{\tilde{\kappa}}{\lambda_{\max }\left(\hat{A}_{l}\right)}, \\
& \tilde{L} \tilde{\Gamma}+\frac{\beta-\alpha}{2} I_{N}+\frac{1}{2(1-\mu)} I_{N}<0, \\
& \beta-\alpha \theta>0, \\
& \alpha \rho-a_{*}>0,
\end{aligned}
$$

where

$$
\begin{aligned}
& \tilde{\kappa}=\frac{1}{\lambda_{\min }(\tilde{\Gamma})}\left(\tilde{L}\|\tilde{\Gamma}\|+\frac{\beta}{2} I_{N}+\frac{1}{2(1-\mu)} I_{N}\right), \\
& \theta=\left(1-\eta_{1}\right) \frac{T_{1}}{T}+\left(1-\eta_{2}\right) \frac{T_{2}}{T}, \quad \rho=\frac{\eta_{1} T_{1}+\eta_{2} T_{2}}{T}, \\
& \hat{A}=\frac{\operatorname{Sym}\{A\}}{2}, \quad a_{*}=\alpha-\beta,
\end{aligned}
$$

$\hat{A}_{l}$ is the minor matrix of $A$ by removing its first $l(1 \leq l \leq N)$ row-column pairs.

Proof The proof is omitted here, as it is similar to that of Theorem 3.1.

\section{Numerical examples and simulation}

In this section, two numerical examples will be provided to verify and demonstrate the effectiveness of the proposed method. 
Example 1 Theorem 3.1 is verified. The node dynamics of the first community are chosen as the well-known Lorenz system:

$$
\left[\begin{array}{c}
\dot{x}_{1} \\
\dot{x}_{2} \\
\dot{x}_{3}
\end{array}\right]=\left[\begin{array}{c}
-10 x_{1}+10 x_{2} \\
28 x_{1}-x_{2}-x_{1} x_{3} \\
-\frac{8}{3} x_{3}+x_{1} x_{2}
\end{array}\right],
$$

and the node dynamics of the second community are chosen as the well-known Chen system:

$$
\left[\begin{array}{c}
\dot{x}_{1} \\
\dot{x}_{2} \\
\dot{x}_{3}
\end{array}\right]=\left[\begin{array}{c}
-35 x_{1}+35 x_{2} \\
-7 x_{1}+28 x_{2}-x_{1} x_{3} \\
-3 x_{3}+x_{1} x_{2}
\end{array}\right],
$$

and the node dynamics of the third community are chosen as the well-known Lv system:

$$
\left[\begin{array}{l}
\dot{x}_{1} \\
\dot{x}_{2} \\
\dot{x}_{3}
\end{array}\right]=\left[\begin{array}{c}
-36 x_{1}+36 x_{2} \\
15 x_{2}-x_{1} x_{3} \\
-3 x_{3}+x_{1} x_{2}
\end{array}\right] .
$$

Similar to the discussion of the Chen system in [24], one can discuss the Lorenz and Lv systems and choose the positive constant $L=65$ such that Assumption 2.1 holds.

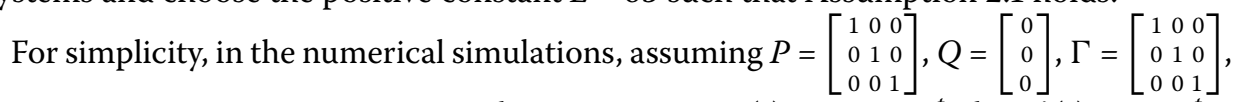
$T_{1}=0.2, T_{2}=0.1, \eta_{1}=0.9, \eta_{2}=0.8$, the time-varying $\tau(t)=1-0.5 e^{-t}$, then $\dot{\tau}(t)=0.5 e^{-t} \in$ $(0,0.5] \leq \frac{1}{2} \doteq \mu<1$. A complex network consisting of 19 nodes with three communities is shown in Figure $2(A=B)$.

The feedback control gain is $k_{i}=20$ for $i \in \bar{V}_{\varphi i}$. By simple calculation, $\theta=\frac{1}{15}, \rho=0.867$, for Theorem 3.1, one can choose $\alpha=155, \beta=22$, and $c<-\frac{\kappa}{\lambda_{\max }\left(\hat{A}_{l}\right)}$ such that conditions (13)-(15) hold. Therefore, the CLGOS can be achieved for any initial values. Figures 3-5 show the orbits of linear generalized outer synchronization errors.

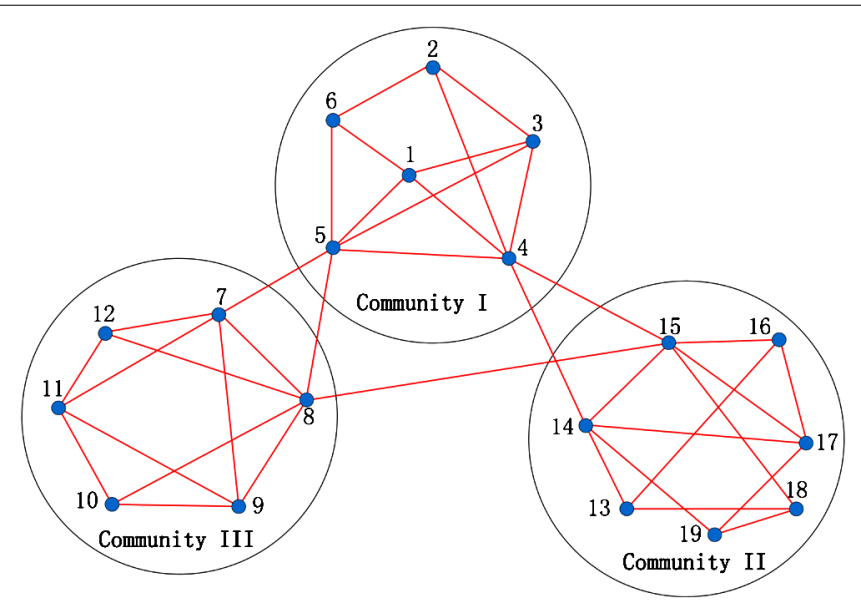

Figure 2 A complex network consisting of 19 nodes with three communities where $a_{i j}=a_{j i}=1$ if there exists a connection between nodes $i$ and $j(j \neq i)$, otherwise $a_{i j}=a_{j i}=0$. 
Figure 3 The orbits of linear generalized outer synchronization errors in the first community.

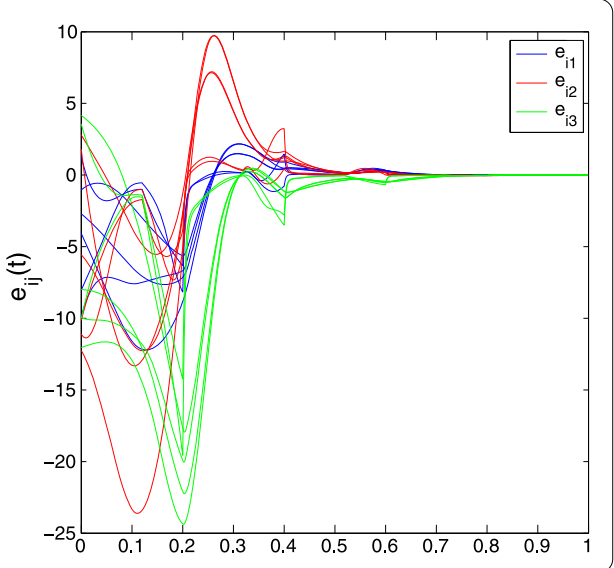

Figure 4 The orbits of linear generalized outer synchronization errors in the second community.

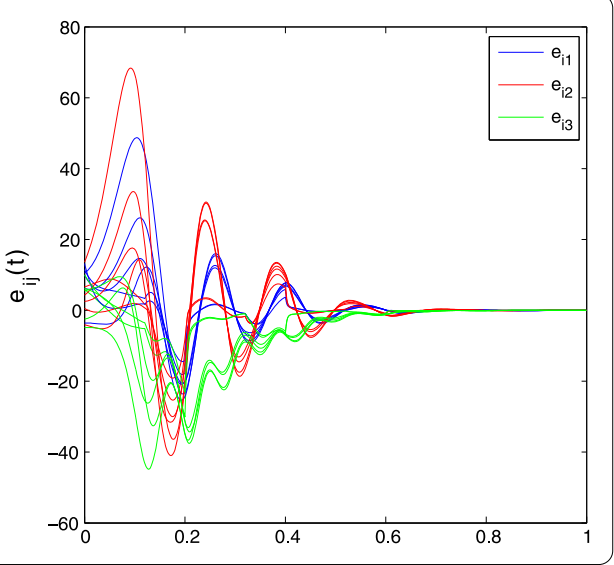

Figure 5 The orbits of linear generalized outer synchronization errors in the third community.

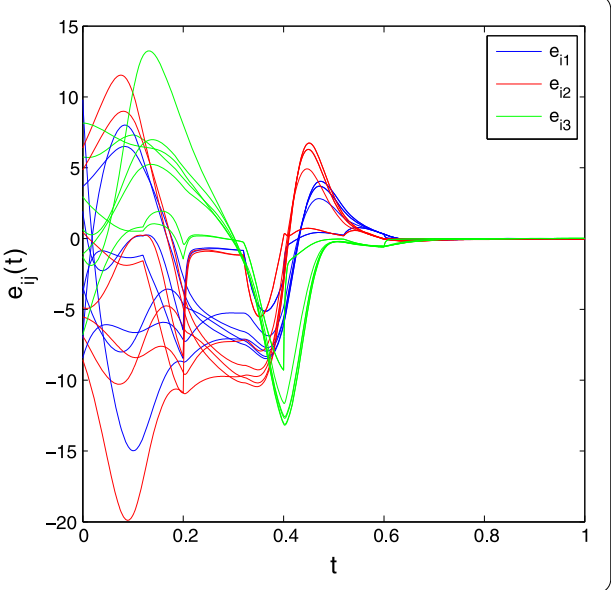

Example 2 Choosing the node dynamics as the following well-known time-delayed Chua oscillator:

$$
\dot{x}(t)=\mathcal{M} x(t)+g_{1}(x(t))+g_{2}(x(t-\tau)),
$$


where

$$
\begin{aligned}
& x(t)=\left(x_{1}(t), x_{2}(t), x_{3}(t)\right)^{T}, \quad \tau=0.02, \\
& \mathcal{M}=\left[\begin{array}{ccc}
-a\left(1+m_{2}\right) & a & 0 \\
1 & -1 & 1 \\
0 & -b & -d
\end{array}\right], \\
& g_{1}(x(t))=\left[\begin{array}{c}
-0.5 a\left(m_{1}-m_{2}\right)\left(\left|x_{1}(t)+1\right|-\left|x_{1}(t)-1\right|\right) \\
0 \\
0
\end{array}\right], \\
& g_{2}(x(t-\tau))=\left[\begin{array}{c}
0 \\
0 \\
-b \chi_{0} \sin \left(v x_{1}(t-\tau)\right)^{T}
\end{array}\right],
\end{aligned}
$$

$a=10, b=19, d=0.1636, m_{1}=-1.4325, m_{2}=-0.7831, v=0.5, \chi_{0}=0.2$. Choose the positive constant $L=12$ such that Assumption 2.1 holds. For simplicity, in the numerical simulations, assuming $P=\left[\begin{array}{lll}1 & 0 & 0 \\ 0 & 1 & 0 \\ 0 & 0 & 1\end{array}\right], Q=\left[\begin{array}{l}0 \\ 0 \\ 0\end{array}\right], \Gamma=\left[\begin{array}{lll}1 & 0 & 0 \\ 0 & 1 & 0 \\ 0 & 0 & 1\end{array}\right], T_{1}=0.2, T_{2}=0.1, \eta_{1}=0.9, \eta_{2}=0.8$, the time-varying $\tau(t)=1-0.5 e^{-t}$, then $\dot{\tau}(t)=0.5 e^{-t} \in(0,0.5] \leq \frac{1}{2} \doteq \mu<1$. A complex network consisting of 19 nodes with three communities is shown in Figure $2(A=B)$. The community network is constructed by integrating three BA networks consisting of 50 nodes with $m_{0}=m=3$. For any pair of communities, four edges are chosen to connect them randomly (Figure 6). The feedback control gains as $k_{i}=20$ for $i \in \bar{V}_{\varphi i}$. By simple calculations, $\theta=\frac{1}{15}, \rho=0.867$, for Theorem 3.1, one can choose $\alpha=35, \beta=5$, and $c<-\frac{\kappa}{\lambda_{\max }\left(\hat{A}_{l}\right)}$ such that condition (13)-(15) hold. Therefore, the CLGOS can be achieved for any initial values. Figures 7-9 show the orbits of linear generalized outer synchronization errors.

\section{Conclusions}

In this paper, we investigated the problems of CLGOS in community networks via pinning control with two different switch periods. Using Lyapunov stability theory, linear matrix inequality (LMI), sufficient CLGOS criteria for community networks are

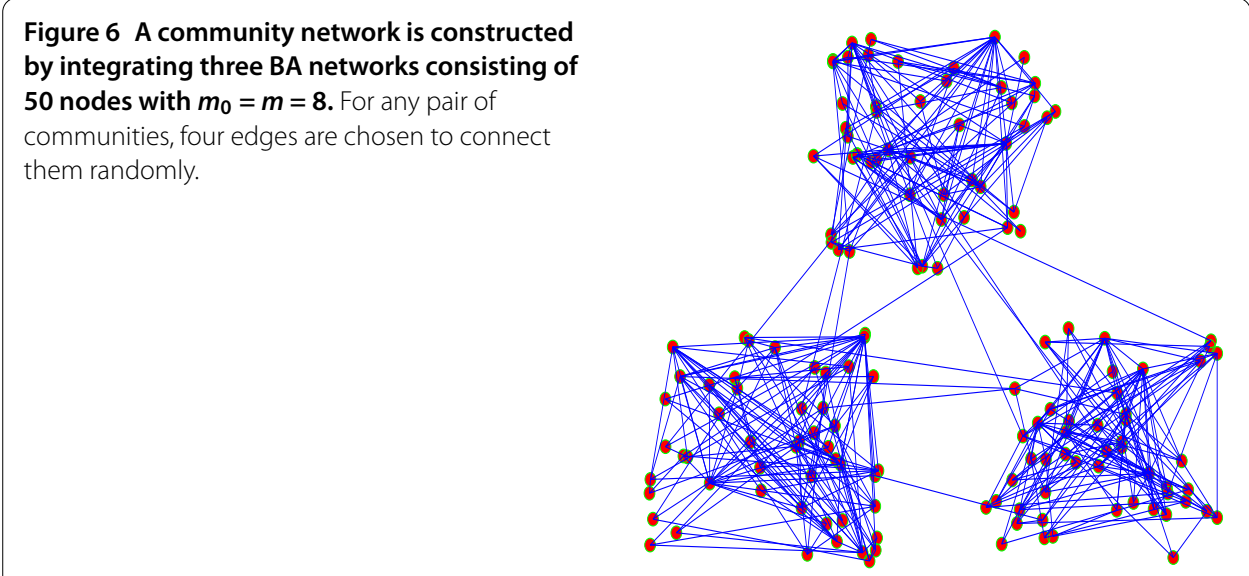




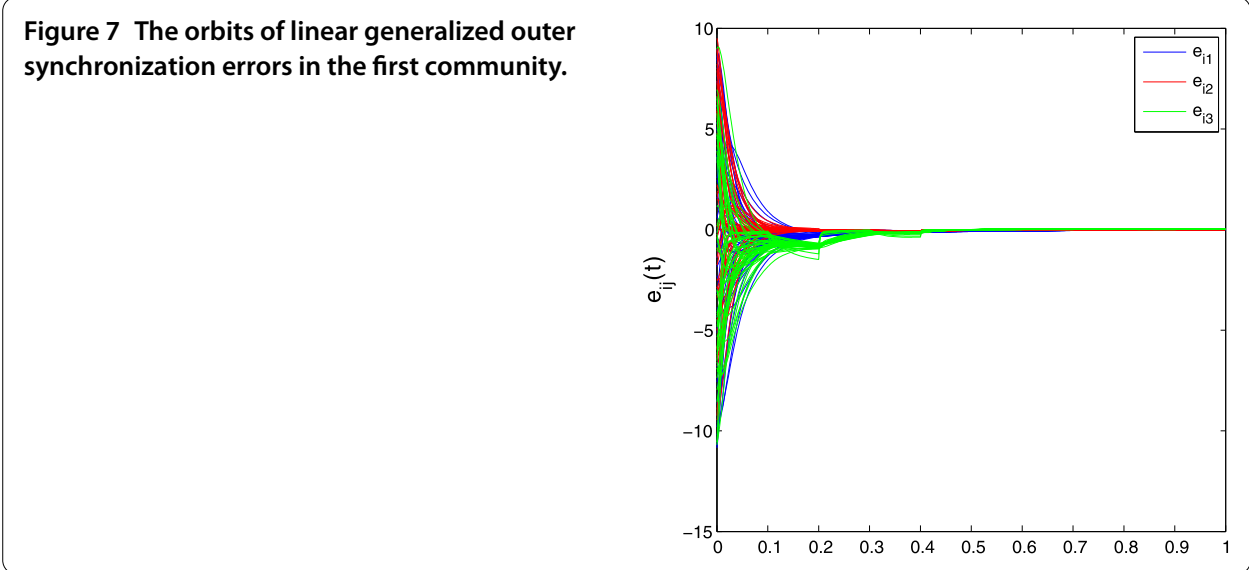

Figure 8 The orbits of linear generalized outer synchronization errors in the second community.

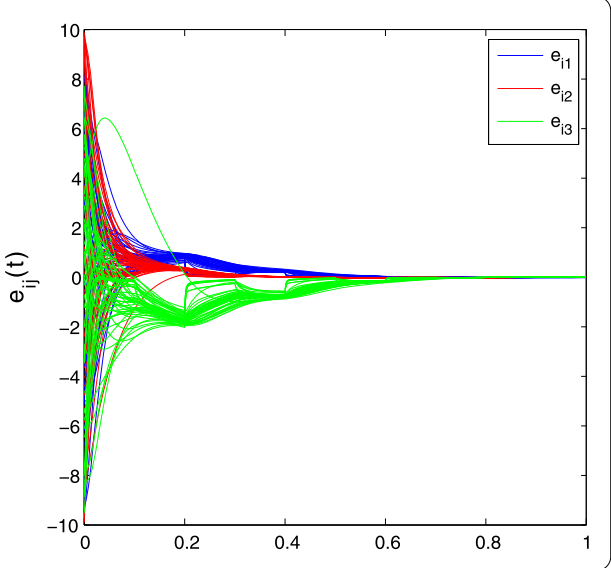

Figure 9 The orbits of linear generalized outer synchronization errors in the third community.

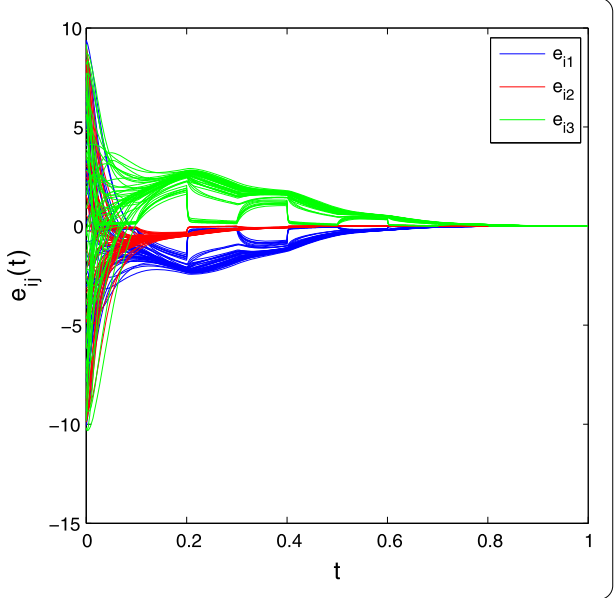

derived. Both community networks with identical nodes and non-identical nodes are investigated. Therefore, our proposed control schemes are better applicable technically. Finally, numerical examples are provided to demonstrate the effectiveness of the proposed method. 


\section{Competing interests}

The authors declare that there is no conflict of interest regarding the publication of this paper.

\section{Authors' contributions}

YHL carried out the main part of this article, corrected and revised the manuscript, HL, SMZ, QSZ brought forward some suggestions on this article. All authors have read and approved the final manuscript.

\section{Author details}

'School of Aeronautics and Astronautics, University of Electronic Science and Technology of China, Chengdu, 611731, China. ${ }^{2}$ School of Mathematical Sciences, University of Electronic Science and Technology of China, Chengdu, 611731, China. ${ }^{3}$ Key Laboratory for Neuroinformation of Ministry of Education, University of Electronic Science and Technology of China, Chengdu, 611731, China.

\section{Acknowledgements}

The authors greatly appreciate the reviewers suggestions and the editors encouragement. The work is partially supported by the Sichuan Science and Technology Plan (2017GZ0165).

\section{Publisher's Note}

Springer Nature remains neutral with regard to jurisdictional claims in published maps and institutional affiliations.

\section{Received: 20 December 2016 Accepted: 6 April 2017 Published online: 20 April 2017}

\section{References}

1. Huang, DW, Yu, ZG, Anh, V: Multifractal analysis and topological properties of a new family of weighted Koch networks. Phys. A, Stat. Mech. Appl. 469, 695-705 (2017)

2. Chen, Y, Wang, X, Xiang, X, Tang, B, Chen, Q, Fan, S, Bu, J: Overlapping community detection in weighted networks via a Bayesian approach. Phys. A, Stat. Mech. Appl. 468, 790-801 (2017)

3. Huang, C, Li, H, Xia, D, Xiao, L: Quantized subgradient algorithm with limited bandwidth communications for solving distributed optimization over general directed multi-agent networks. Neurocomputing 185, 153-162 (2016)

4. Wang, X, Yang, GH: Distributed consensus tracking control for multi-agent networks with switching directed topologies. Neurocomputing 207, 693-699 (2016)

5. $\mathrm{Hu}, \mathrm{X}, \mathrm{Li}, \mathrm{Y}, \mathrm{Xu}, \mathrm{H}$ : Multi-mode clustering model for hierarchical wireless sensor networks. Phys. A, Stat. Mech. Appl. 469, 665-675 (2017)

6. Yang, L, Jiang, J, Liu, X: Cluster synchronization in community network with hybrid coupling. Chaos Solitons Fractals 86, 82-91 (2016)

7. Kim, P, Kim, S: Detecting community structure in complex networks using an interaction optimization process. Phys. A, Stat. Mech. Appl. 465, 525-542 (2017)

8. Chen, Y, Wang, X, Xiang, X, Tang, B, Chen, Q, Fan, S, Bu, J: Overlapping community detection in weighted networks via a Bayesian approach. Phys. A, Stat. Mech. Appl. 468, 790-801 (2017)

9. Kaviarasan, B, Sakthivel, R, Lim, Y: Synchronization of complex dynamical networks with uncertain inner coupling and successive delays based on passivity theory. Neurocomputing 186, 127-138 (2016)

10. Li, WL, Zhang, FY, Li, C, Song, HS: Quantum synchronization in a star-type cavity QED network. Commun. Nonlinear Sci. Numer. Simul. 42, 121-131 (2017)

11. Sakthivel, R, Anbuvithya, R, Mathiyalagan, K, Ma, YK, Prakash, P: Reliable anti-synchronization conditions for BAM memristive neural networks with different memductance functions. Appl. Math. Comput. 275, 213-228 (2016)

12. Thuan, MV, Trinh, H, Hien, LV: New inequality-based approach to passivity analysis of neural networks with interval time-varying delay. Neurocomputing 194, 301-307 (2016)

13. Ray, A, Roychowdhury, A: Outer synchronization of networks with different node dynamics. Eur. Phys. J. Spec. Top. 223, 1509-1518 (2014)

14. Ahmadizadeh, S, Freestone, DR, Grayden, DB: On synchronization of networks of Wilson-Cowan oscillators with diffusive coupling. Automatica 71, 169-178 (2016)

15. Celikovsky, S, Lynnyk, V, Chen, G: Robust synchronization of a class of chaotic networks. J. Franklin Inst. 350 2936-2948 (2013)

16. Franceschelli, M, Giua, A, Pisano, A, Usai, E: Finite-time consensus for switching network topologies with disturbances. Nonlinear Anal. Hybrid Syst. 10, 83-93 (2013)

17. Li, WL, Li, C, Song, HS: Synchronization between uncertain nonidentical networks with quantum chaotic behavior Physica A 461, 270-277 (2016)

18. Zhou, GY, Li, CR, Li, TT, Yang, Y, Wang, C, He, FJ, Sun, JC: Outer synchronization investigation between WS and NW small-world networks with different node numbers. Physica A 457, 506-513 (2016)

19. Rakkiyappan, R, Sakthivel, N: Pinning sampled-data control for synchronization of complex networks with probabilistic time-varying delays using quadratic convex approach. Neurocomputing 162, 26-40 (2015)

20. Lü, L, Chen, LS, Bai, SY, Li, G: A new synchronization tracking technique for uncertain discrete network with spatiotemporal chaos behaviors. Physica A 460, 314-325 (2016)

21. Li, WL, Li, C, Song, HS: Synchronization transmission of target signal within the coupling network with quantum chaos effect. Physica A 462, 579-585 (2016)

22. Skardal, PS, Taylor, D, Sun, J, Arenas, A: Erosion of synchronization in networks of coupled oscillators. Phys. Rev. E 91(1), $010802(2015)$

23. Dörfler, F, Bullo, F: Synchronization in complex networks of phase oscillators: a survey. Automatica 50, 1539-1564 (2014)

24. Al-Mahbashi, G, Noorani, MSM, Bakar, SA: Projective lag synchronization in drive-response dynamical networks. Int. J. Mod. Phys. C 25, 771-776 (2014) 
25. Bao, HB, Cao, JD: Projective synchronization of fractional-order memristor-based neural networks. Neural Netw. 63(3), 1-9 (2015)

26. Jalan, S, Singh, A, Acharyya, S, Kurths, J: Impact of a leader on cluster synchronization. Phys. Rev. E 91(2), 022901 (2015)

27. Hou, HZ, Zhang, QL, Zheng, M: Cluster synchronization in nonlinear complex networks under sliding mode control. Nonlinear Dyn. 83(1-2), 739-749 (2016)

28. Rakkiyappan, R, Sakthivel, N: Cluster synchronization for T-S fuzzy complex networks using pinning control with probabilistic time-varying delays. Complexity 21(1), 59-77 (2015)

29. Cui, G, Zhuang, G, Lu, J: Neural-network-based distributed adaptive synchronization for nonlinear multi-agent systems in pure-feedback form. Neurocomputing 218, 234-241 (2016)

30. Xu, Y, Zhang, J, Zhou, W, Tong, D: Adaptive synchronization of complex dynamical networks with bounded delay feedback controller. Optik 131, 467-474 (2017)

31. Wei, W, Wang, M, Li, D, Zuo, M, Wang, X: Disturbance observer based active and adaptive synchronization of energy resource chaotic system. ISA Trans. 65, 164-173 (2016)

32. Zhang, WY, Yang, C, Guan, ZH, Liu, ZW, Chi, M, Zheng, GL: Bounded synchronization of coupled Kuramoto oscillators with phase lags via distributed impulsive control. Neurocomputing 218, 216-222 (2016)

33. Feng, J, Yang, P, Zhao, Y: Cluster synchronization for nonlinearly time-varying delayed coupling complex networks with stochastic perturbation via periodically intermittent pinning control. Appl. Math. Comput. 291, 52-68 (2016)

34. Wen, GH, Duan, ZS, Chen, GR, Yu, WW: Consensus tracking of multi-agent systems with Lipschitz-type node dynamics and switching topologies. IEEE Trans. Circuits Syst. 61(2), 499-511 (2014)

35. Wen, GH, Yu, WW, Hu, GH, Cao, GD, Yu, XH: Pinning synchronization of directed networks with switching topologies: a multiple Lyapunov functions approach. IEEE Trans. Neural Netw. Learn. Syst. 26(12), 3239-3250 (2015)

36. Lei, X, Cai, S, Jiang, S, Liu, Z: Adaptive outer synchronization between two complex delayed dynamical networks via aperiodically intermittent pinning control. Neurocomputing 222, 26-35 (2017)

37. Song, Q, Cao, J: On pinning synchronization of directed and undirected complex dynamical networks. IEEE Trans. Circuits Syst. I 57, 672-680 (2010)

38. Li, CP, Sun, WG, Kurths, J: Synchronization between two coupled complex networks. Phys. Rev. E 76, 046204 (2007)

39. Tang, HW, Chen, L, Lu, JA, Tse, CK: Adaptive synchronization between two complex networks with nonidentical topological structures. Physica A 387, 5623-5630 (2008)

40. Zheng, S, Bi, Q, Cai, G: Adaptive projective synchronization between two complex networks with time-varying coupling delay. Phys. Lett. A 373, 1553-1559 (2009)

41. Wu, X, Zheng, W, Zhou, J: Generalized outer synchronization between complex dynamical networks. Chaos 19 $013109(2009)$

42. Sun, M, Zeng, CY, Tian, LX: Linear generalized synchronization between two complexes. Commun. Nonlinear Sci. Numer. Simul. 15, 2162-2167 (2010)

\section{Submit your manuscript to a SpringerOpen ${ }^{\ominus}$ journal and benefit from:}

- Convenient online submission

- Rigorous peer review

- Immediate publication on acceptance

Open access: articles freely available online

- High visibility within the field

- Retaining the copyright to your article 